\title{
Signez maintenant pour la protection contre le tabagisme passif!
}

\author{
En automne 2008, les Chambres fédérales ont adopté une loi fédérale qui protège \\ insuffisamment la population contre le tabagisme passif. C'est pourquoi plus de \\ 45 associations, dont de nombreuses sociétés médicales, se sont unies pour lancer, \\ le 25 mai 2009, I'initiative populaire «Protection contre le tabagisme passif». Cette \\ initiative demande une protection complète pour tous les travailleurs et une régle- \\ mentation uniforme pour toute la Suisse.
}

\section{Werner Karrer}

Médecin-chef à la Clinique d'altitude lucernoise de Montana, Président de la Société Suisse de Pneumologie, Membre du Comité d'initiative de l'initiative populaire «Protection contre le tabagisme passif»

Correspondance:

Dr Werner Karrer

Clinique d'altitude lucernoise

de Montana

CH-3963 Crans-Montana

Tél. 0274858151

Fax 0274817364

werner.karrer@LHM.ch
Il est prouvé depuis de nombreuses années que le tabagisme passif est dangereux. En Suisse aussi, sa nocivité a fait l'objet de recherches, notamment dans l'étude SAPALDIA ( «Swiss Study on Air Pollution And Lung Diseases In Adults»). Le danger que représente la fumée passive est attesté scientifiquement [1].

Les plus touchés sont les employés de la restauration. Ils et elles sont plus de 100000 à être exposés pendant toute la journée à la fumée du tabac. En Suisse, il n'existe aucune autre profession exposée à un risque comparable aussi facilement évitable. Des mesures de poussières fines dans les établissements de restauration suisses ne laissent pas prise au doute: la santé de toute personne travaillant dans un local fumeur est menacée [2]. C'est pourquoi il va de soi aujourd'hui dans la plupart des pays d'Europe que les lieux de travail sont sans fumée - y compris dans la

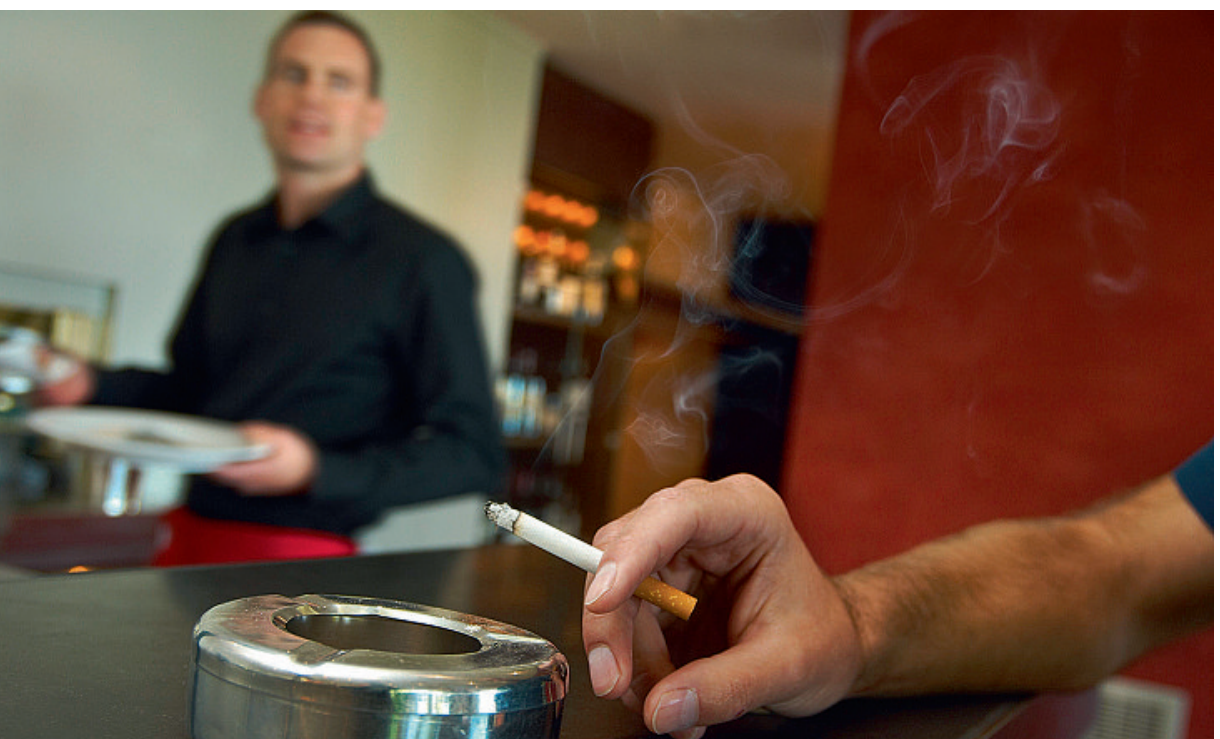

Fumer pendant que les autres mangent sera peut-être bientôt interdit.

restauration. Les effets positifs sur la santé qu'entraîne un environnement de travail sans fumée se manifestent déjà au bout de très peu de temps, comme des études scientifiques l'ont montré. Le passage à une restauration sans fumée conduit à

- une réduction drastique des particules pouvant pénétrer dans les poumons ainsi que des substances susceptibles de provoquer le cancer ou de nuire au patrimoine génétique;

- une amélioration de la fonction pulmonaire des collaborateurs non-fumeurs;

- une amélioration de la santé des employés nonfumeurs et fumeurs: nettement moins de problèmes des voies respiratoires, d'irritations des muqueuses, de rougeurs aux yeux, de toux.

\section{L'initiative est seule à proposer une protection efficace}

Des réglementations conséquentes et générales sur la fumée ont des effets positifs sur la santé de toute la population. Sitôt que des exceptions viennent diluer la protection contre le tabagisme passif - comme c'est le cas dans la Loi fédérale adoptée en automne 2008 - ces effets positifs sur la santé ne peuvent plus être escomptés, du moins plus dans des proportions aussi réjouissantes.

La Loi fédérale sur la protection contre le tabagisme passif que les Chambres fédérales ont adoptée le 3 octobre 2008 ne mérite pas son nom. Les établissements dont la surface ne dépasse pas $80 \mathrm{~m}^{2}$ peuvent rester fumeurs, et tous les établissements ont le droit d'aménager des fumoirs. Dans les deux cas, les serveuses ou serveurs sont insuffisamment protégés de la fumée du tabac. En outre, les réglementations sur la fumée continueront de différer d'un canton à l'autre.

L'initiative populaire fédérale demande une réglementation uniforme dans toute la Suisse et efficace pour protéger la population contre le tabagisme pas- 
